\title{
On a Fast Convergence of the Rational-Trigonometric-Polynomial Interpolation
}

\author{
Arnak Poghosyan \\ Institute of Mathematics, National Academy of Sciences, Marshal Baghramian Avenue 24b, 0019 Yerevan, Armenia \\ Correspondence should be addressed to Arnak Poghosyan; arnak@instmath.sci.am
}

Received 11 October 2012; Revised 8 January 2013; Accepted 20 January 2013

Academic Editor: Hassan Safouhi

Copyright (C) 2013 Arnak Poghosyan. This is an open access article distributed under the Creative Commons Attribution License, which permits unrestricted use, distribution, and reproduction in any medium, provided the original work is properly cited.

We consider the convergence acceleration of the Krylov-Lanczos interpolation by rational correction functions and investigate convergence of the resultant parametric rational-trigonometric-polynomial interpolation. Exact constants of asymptotic errors are obtained in the regions away from discontinuities, and fast convergence of the rational-trigonometric-polynomial interpolation compared to the Krylov-Lanczos interpolation is observed. Results of numerical experiments confirm theoretical estimates and show how the parameters of the interpolations can be determined in practice.

\section{Introduction}

In this paper, we continue investigations started in [1] where we considered the convergence acceleration of the classical trigonometric interpolation

$$
\begin{gathered}
I_{N}(f, x)=\sum_{n=-N}^{N} \check{f}_{n} e^{i \pi n x}, \\
\check{f}_{n}=\frac{1}{2 N+1} \sum_{k=-N}^{N} f\left(x_{k}\right) e^{-i \pi n x_{k}}, \quad x_{k}=\frac{2 k}{2 N+1},
\end{gathered}
$$

via polynomial corrections representing discontinuities of the function $f$ and some of its first $q$ derivatives (jumps). The resultant interpolation $I_{N, q}(f, x)$ was called as the KrylovLanczos (KL-) interpolation. That approach was suggested in 1906 by Krylov [2] and later in 1964 by Lanczos [3, 4] (see also [1,5-10] with references therein).

Here, we consider the convergence acceleration of the KLinterpolation by the application of rational (by $e^{i \pi x}$ ) correction functions along the ideas of the rational approximations (see [11-13] with references therein). The approach discussed here leads to the parametric (depending on parameters $\left.\theta_{1}, \ldots, \theta_{p}\right)$ rational-trigonometric-polynomial (rtp-) interpolation $I_{N, q}^{p}(f, x)$. The idea of the convergence acceleration via sequential application of polynomial and rational corrections was described in [14-17]. The KL-interpolation is a special case of the rtp-interpolation corresponding to the choice of parameters $\theta_{k}=0, k=1, \ldots, p$. Besides, rational corrections can be applied immediately to the classical interpolation without polynomial corrections (see interpolation $I_{N}^{p}(f, x)$ ).

In this paper, we reveal the convergence properties of the rtp-interpolation, show its fast convergence compared to the KL-interpolation in the regions away from the singularities $(x= \pm 1)$, and discuss the problem of parameters determination in rational corrections.

\section{Rational Interpolations}

In this section, we introduce a rational interpolation as a method of the convergence acceleration of the classical trigonometric interpolation. Here, we recap some details from $[15,16]$.

By $r_{N}(f, x)$, we denote the error of the classical trigonometric interpolation

$$
r_{N}(f, x)=f(x)-I_{N}(f, x)
$$


and write

$$
\begin{aligned}
r_{N}(f, x)= & \sum_{n=-N}^{N}\left(f_{n}-\check{f}_{n}\right) e^{i \pi n x}+\sum_{n=N+1}^{\infty} f_{n} e^{i \pi n x} \\
& +\sum_{n=-\infty}^{-N-1} f_{n} e^{i \pi n x},
\end{aligned}
$$

where $f_{n}$ is the $n$th Fourier coefficient of $f$

$$
f_{n}=\frac{1}{2} \int_{-1}^{1} f(x) e^{-i \pi n x} d x
$$

Rational corrections considered in this paper are based on a series of formulae of summation by parts applied to the error terms in (4). Such transformations lead to new interpolations with correction terms in the form of rational (by $e^{i \pi x}$ ) functions. Consider a vector of complex numbers $\theta=\left\{\theta_{1}, \ldots, \theta_{p}\right\}$. The first formula of summation by parts is easy to verify straightforwardly as

$$
\begin{aligned}
\sum_{n=N+1}^{\infty} f_{n} e^{i \pi n x}= & -\theta_{1} f_{N} \frac{e^{i \pi(N+1) x}}{1+\theta_{1} e^{i \pi x}} \\
& +\frac{1}{1+\theta_{1} e^{i \pi x}} \sum_{n=N+1}^{\infty}\left(f_{n}+\theta_{1} f_{n-1}\right) e^{i \pi n x} .
\end{aligned}
$$

If here $\left|\theta_{1}\right|<1$, then the formula is valid for all $|x| \leq 1$. This is the main reason of including the parameters $\theta_{k}$ here and further. If $\theta_{1}$ is chosen appropriately (see (32)), then the second term in the right-hand side of (6) converges faster (however, for $|x|<1$ ) than the sum in the left-hand side. The next, slightly different formula of summation by parts is also easy to derive as

$$
\begin{aligned}
\sum_{n=N+1}^{\infty} f_{n} e^{i \pi n x}= & \theta_{1} f_{N+1} \frac{e^{i \pi N x}}{1+\theta_{1} e^{-i \pi x}} \\
& +\frac{1}{1+\theta_{1} e^{-i \pi x}} \sum_{n=N+1}^{\infty}\left(f_{n}+\theta_{1} f_{n+1}\right) e^{i \pi n x}
\end{aligned}
$$

Application of (7) to the second term in the right-hand side of (6) leads to the needed expansion

$$
\begin{aligned}
\sum_{n=N+1}^{\infty} f_{n} e^{i \pi n x} & \\
= & \frac{1}{\left(1+\theta_{1} e^{i \pi x}\right)\left(1+\theta_{1} e^{-i \pi x}\right)} \\
& \times \sum_{n=N+1}^{\infty}\left(f_{n}+\theta_{1} f_{n-1}+\theta_{1}\left(f_{n+1}+\theta_{1} f_{n}\right)\right) e^{i \pi n x} \\
& -\theta_{1} f_{N} \frac{e^{i \pi(N+1) x}}{\left(1+\theta_{1} e^{i \pi x}\right)\left(1+\theta_{1} e^{-i \pi x}\right)} \\
& +\theta_{1} f_{N+1} \frac{e^{i \pi N x}}{\left(1+\theta_{1} e^{i \pi x}\right)\left(1+\theta_{1} e^{-i \pi x}\right)} .
\end{aligned}
$$

Here, also, as we mentioned above, the sum on the right-hand side converges faster than on the left-hand side if parameter $\theta_{1}$ is chosen appropriately.

In a similar manner, we transform the third term in the right-hand side of (4):

$$
\begin{aligned}
\sum_{n=-\infty}^{-N-1} f_{n} e^{i \pi n x} & \\
= & \frac{1}{\left(1+\theta_{1} e^{i \pi x}\right)\left(1+\theta_{1} e^{-i \pi x}\right)} \\
& \times \sum_{n=-\infty}^{-N-1}\left(f_{n}+\theta_{1} f_{n-1}+\theta_{1}\left(f_{n+1}+\theta_{1} f_{n}\right)\right) e^{i \pi n x} \\
& -\theta_{1} f_{-N} \frac{e^{-i \pi(N+1) x}}{\left(1+\theta_{1} e^{i \pi x}\right)\left(1+\theta_{1} e^{-i \pi x}\right)} \\
& +\theta_{1} f_{-N-1} \frac{e^{-i \pi N x}}{\left(1+\theta_{1} e^{i \pi x}\right)\left(1+\theta_{1} e^{-i \pi x}\right)} .
\end{aligned}
$$

For the first term in the right-hand side of (4), the formula of summation by parts is the following:

$$
\begin{aligned}
\sum_{n=-N}^{N} c_{n} e^{i \pi n x}= & \frac{1}{\left(1+\theta_{1} e^{i \pi x}\right)\left(1+\theta_{1} e^{-i \pi x}\right)} \\
& \times \sum_{n=-N}^{N}\left(c_{n}+\theta_{1} c_{n-1}+\theta_{1}\left(c_{n+1}+\theta_{1} c_{n}\right)\right) e^{i \pi n x} \\
& +\theta_{1} c_{N} \frac{e^{i \pi(N+1) x}}{\left(1+\theta_{1} e^{i \pi x}\right)\left(1+\theta_{1} e^{-i \pi x}\right)} \\
& -\theta_{1} c_{-N-1} \frac{e^{-i \pi N x}}{\left(1+\theta_{1} e^{i \pi x}\right)\left(1+\theta_{1} e^{-i \pi x}\right)} \\
& -\theta_{1} c_{N+1} \frac{e^{i \pi N x}}{\left(1+\theta_{1} e^{i \pi x}\right)\left(1+\theta_{1} e^{-i \pi x}\right)} \\
& +\theta_{1} c_{-N} \frac{e^{-i \pi(N+1) x}}{\left(1+\theta_{1} e^{i \pi x}\right)\left(1+\theta_{1} e^{-i \pi x}\right)}
\end{aligned}
$$

where $c_{n}=f_{n}-\check{f}_{n}$.

Substituting (8), (9), and (10) into (4), after some simplifications, provides with the following expansion of the error

$$
\begin{aligned}
r_{N}(f, x)= & \theta_{1} \check{f}_{N} \frac{e^{-i \pi N x}-e^{i \pi(N+1) x}}{\left(1+\theta_{1} e^{i \pi x}\right)\left(1+\theta_{1} e^{-i \pi x}\right)} \\
& +\theta_{1} \check{f}_{-N} \frac{e^{i \pi N x}-e^{-i \pi(N+1) x}}{\left(1+\theta_{1} e^{i \pi x}\right)\left(1+\theta_{1} e^{-i \pi x}\right)}
\end{aligned}
$$




$$
\begin{aligned}
& +\frac{1}{\left(1+\theta_{1} e^{i \pi x}\right)\left(1+\theta_{1} e^{-i \pi x}\right)} \\
& \times \sum_{|n|=N+1}^{\infty}\left(f_{n}+\theta_{1} f_{n-1}+\theta_{1}\left(f_{n+1}+\theta_{1} f_{n}\right)\right) e^{i \pi n x} \\
& +\frac{1}{\left(1+\theta_{1} e^{i \pi x}\right)\left(1+\theta_{1} e^{-i \pi x}\right)} \\
& \times \sum_{n=-N}^{N}\left(c_{n}+\theta_{1} c_{n-1}+\theta_{1}\left(c_{n+1}+\theta_{1} c_{n}\right)\right) e^{i \pi n x},
\end{aligned}
$$

where $c_{n}=f_{n}-\check{f}_{n}$. Here, we also took into account the periodicity of the coefficients $\check{f}_{n}$

$$
\check{f}_{N+1}=\check{f}_{-N}, \quad \check{f}_{-N-1}=\check{f}_{N} \text {. }
$$

For writing the expansion (11) in a short form and also for further reiterations of this transformation, we introduce the following generalized finite differences $\delta_{n}^{k}\left(\theta, y_{n}\right)$ determined recurrently:

$$
\begin{gathered}
\delta_{n}^{0}\left(\theta, y_{n}\right)=y_{n} \\
\delta_{n}^{k}\left(\theta, y_{n}\right)=\delta_{n}^{k-1}\left(\theta, y_{n}\right)+\theta_{k} \delta_{n-1}^{k-1}\left(\theta, y_{n}\right) \\
+\theta_{k}\left(\delta_{n+1}^{k-1}\left(\theta, y_{n}\right)+\theta_{k} \delta_{n}^{k-1}\left(\theta, y_{n}\right)\right),
\end{gathered}
$$

for some sequence $y_{n}$. Now, (11) can be rewritten in the form

$$
\begin{aligned}
r_{N}(f, x)= & \theta_{1} \delta_{N}^{0}\left(\theta, \check{f}_{n}\right) \frac{e^{-i \pi N x}-e^{i \pi(N+1) x}}{\left(1+\theta_{1} e^{i \pi x}\right)\left(1+\theta_{1} e^{-i \pi x}\right)} \\
& +\theta_{1} \delta_{-N}^{0}\left(\theta, \check{f}_{n}\right) \frac{e^{i \pi N x}-e^{-i \pi(N+1) x}}{\left(1+\theta_{1} e^{i \pi x}\right)\left(1+\theta_{1} e^{-i \pi x}\right)} \\
& +\frac{1}{\left(1+\theta_{1} e^{i \pi x}\right)\left(1+\theta_{1} e^{-i \pi x}\right)} \\
& \times \sum_{|n|=N+1}^{\infty} \delta_{n}^{1}\left(\theta, f_{n}\right) e^{i \pi n x} \\
& +\frac{1}{\left(1+\theta_{1} e^{i \pi x}\right)\left(1+\theta_{1} e^{-i \pi x}\right)} \\
& \times \sum_{n=-N}^{N} \delta_{n}^{1}\left(\theta, f_{n}-\check{f}_{n}\right) e^{i \pi n x} .
\end{aligned}
$$

Reiteration of this transformation up to $p$ times leads to the following expansion of the error:

$$
\begin{aligned}
r_{N}( & f, x) \\
= & \left(e^{-i \pi N x}-e^{i \pi(N+1) x}\right) \\
& \times \sum_{k=1}^{p} \frac{\theta_{k} \delta_{N}^{k-1}\left(\theta, \check{f}_{n}\right)}{\prod_{s=1}^{k}\left(1+\theta_{s} e^{i \pi x}\right)\left(1+\theta_{s} e^{-i \pi x}\right)} \\
& +\left(e^{i \pi N x}-e^{-i \pi(N+1) x}\right) \\
& \times \sum_{k=1}^{p} \frac{\theta_{k} \delta_{-N}^{k-1}\left(\theta, \check{f}_{n}\right)}{\prod_{s=1}^{k}\left(1+\theta_{s} e^{i \pi x}\right)\left(1+\theta_{s} e^{-i \pi x}\right)} \\
& +\frac{1}{\prod_{s=1}^{p}\left(1+\theta_{s} e^{i \pi x}\right)\left(1+\theta_{s} e^{-i \pi x}\right)} \sum_{|n|=N+1}^{\infty} \delta_{n}^{p}\left(\theta, f_{n}\right) e^{i \pi n x} \\
& +\frac{1}{\prod_{s=1}^{p}\left(1+\theta_{s} e^{i \pi x}\right)\left(1+\theta_{s} e^{-i \pi x}\right)} \\
& \times \sum_{n=-N}^{N} \delta_{n}^{p}\left(\theta, f_{n}-\check{f}\right) e_{n}^{i \pi n x},
\end{aligned}
$$

where the first two terms in the right-hand side can be viewed as rational corrections to the error and the last two terms as the actual error. This observation leads to the following rational-trigonometric interpolation:

$$
\begin{aligned}
I_{N}^{p}(f, x)= & I_{N}(f, x)+\left(e^{-i \pi N x}-e^{i \pi(N+1) x}\right) \\
& \times \sum_{k=1}^{p} \frac{\theta_{k} \delta_{N}^{k-1}\left(\theta, \check{f}_{n}\right)}{\prod_{s=1}^{k}\left(1+\theta_{s} e^{i \pi x}\right)\left(1+\theta_{s} e^{-i \pi x}\right)} \\
& +\left(e^{i \pi N x}-e^{-i \pi(N+1) x}\right) \\
& \times \sum_{k=1}^{p} \frac{\theta_{k} \delta_{-N}^{k-1}\left(\theta, \check{f}_{n}\right)}{\prod_{s=1}^{k}\left(1+\theta_{s} e^{i \pi x}\right)\left(1+\theta_{s} e^{-i \pi x}\right)},
\end{aligned}
$$

with the error

$$
\begin{aligned}
r_{N}^{p}(f, x)= & \frac{1}{\prod_{s=1}^{p}\left(1+\theta_{s} e^{i \pi x}\right)\left(1+\theta_{s} e^{-i \pi x}\right)} \\
& \times \sum_{|n|=N+1}^{\infty} \delta_{n}^{p}\left(\theta, f_{n}\right) e^{i \pi n x} \\
& +\frac{1}{\prod_{s=1}^{p}\left(1+\theta_{s} e^{i \pi x}\right)\left(1+\theta_{s} e^{-i \pi x}\right)} \\
& \times \sum_{n=-N}^{N} \delta_{n}^{p}\left(\theta, f_{n}-\check{f}_{n}\right) e^{i \pi n x} .
\end{aligned}
$$

The problem of the determination of parameters $\theta_{k}$ will be discussed later. 


\section{The KL-Interpolation}

In this section, we consider the additional acceleration of the rational-trigonometric interpolation by the polynomial correction method known as the Krylov-Lanczos approach. We recap the main ideas from [1].

Let $f \in C^{q-1}[-1,1]$. By $A_{k}(f)$, we denote the jumps of $f$ at the end points of the interval

$$
A_{k}(f)=f^{(k)}(1)-f^{(k)}(-1), \quad k=0, \ldots, q-1 .
$$

The polynomial correction method is based on the following representation of the interpolated function:

$$
f(x)=\sum_{k=0}^{q-1} A_{k}(f) B_{k}(x)+F(x),
$$

where $B_{k}$ are 2-periodic Bernoulli polynomials

$$
\begin{gathered}
B_{0}(x)=\frac{x}{2}, \quad B_{k}(x)=\int B_{k-1}(x) d x, \\
\int_{-1}^{1} B_{k}(x) d x=0, \quad x \in[-1,1],
\end{gathered}
$$

with the Fourier coefficients

$$
B_{n}(k)=\frac{(-1)^{n+1}}{2(i \pi n)^{k+1}}, \quad n \neq 0, B_{0}(k)=0 .
$$

Function $F$ is a 2-periodic and relatively smooth function on the real line $\left(F \in C^{q-1}(R)\right)$ with the discrete Fourier coefficients

$$
\check{F}_{n}=\check{f}_{n}-\sum_{k=0}^{q-1} A_{k}(f) \check{B}_{n}(k) .
$$

The approximation of $F$ in (19) by the classical trigonometric interpolation leads to the Krylov-Lanczos (KL-) interpolation

$$
I_{N, q}(f, x)=\sum_{k=0}^{q-1} A_{k}(f) B_{k}(x)+I_{N}(F, x),
$$

and the approximation of $F$ by the rational-trigonometric interpolation leads to the rational-trigonometric-polynomial (rtp-) interpolation

$$
I_{N, q}^{p}(f, x)=\sum_{k=0}^{q-1} A_{k}(f) B_{k}(x)+I_{N}^{p}(F, x)
$$

with the errors

$$
\begin{aligned}
& r_{N, q}(f, x)=f(x)-I_{N, q}(f, x), \\
& r_{N, q}^{p}(f, x)=f(x)-I_{N, q}^{p}(f, x),
\end{aligned}
$$

respectively.

We need the next results for further comparisons. Theorems 1 and 2 show the behavior of the KL-interpolation in the regions away from the singularities $(x= \pm 1)$.

Denote

$$
\phi_{m}=\sum_{s=-\infty}^{\infty} \frac{(-1)^{s}}{(2 s+1)^{m}} .
$$

Theorem 1 (see [1]). Let $q \geq 2$ be even, $f \in C^{q+1}[-1,1]$, and $f^{(q+1)} \in A C[-1,1]$. Then, the following estimate holds for $|x|<$ 1 ,

$$
r_{N, q}(f, x)=\frac{\varphi_{N, q}(f, x)}{N^{q+1}}+o\left(N^{-q-1}\right), \quad N \longrightarrow \infty,
$$

where

$$
\varphi_{N, q}(f, x)=A_{q}(f) \frac{(-1)^{N+q / 2}}{2 \pi^{q+1}} \frac{\sin (\pi(2 N+1) x / 2)}{\cos (\pi x / 2)} \phi_{q+1} .
$$

Theorem 2 (see [1]). Let $q \geq 1$ be odd, $f \in C^{q+2}[-1,1]$, and $f^{(q+2)} \in A C[-1,1]$. Then, the following estimate holds for $|x|<$ 1 ,

$$
r_{N, q}(f, x)=\frac{\varphi_{N, q}(f, x)}{N^{q+2}}+o\left(N^{-q-2}\right), \quad N \longrightarrow \infty,
$$

where

$$
\begin{aligned}
\varphi_{N, q} & (f, x) \\
= & A_{q}(f) \frac{(-1)^{N+(q+1) / 2+1}(q+1)}{4 \pi^{q+1}} \\
& \times \frac{\sin (\pi x / 2) \sin (\pi(2 N+1) x / 2)}{\cos ^{2}(\pi x / 2)} \phi_{q+2} \\
& +A_{q+1}(f) \frac{(-1)^{N+(q+1) / 2}}{2 \pi^{q+2}} \frac{\sin (\pi(2 N+1) x / 2)}{\cos (\pi x / 2)} \phi_{q+2} .
\end{aligned}
$$

The aim of this paper is the derivation of analogs of Theorems 1 and 2 for the rtp-interpolations.

The determination of parameters $\theta_{k}$ is crucial for the realization of the rtp-interpolation. General method leads to the Fourier-Padé interpolation (see [16]).

Here, we consider a smooth function $f$ on $[-1,1]$ and take

$$
\theta_{k}=1-\frac{\tau_{k}}{N}, \quad k=1, \ldots, p
$$

where the new parameters $\tau_{k}$ are independent of $N$. They can be determined differently. One approach leads to the $L_{2}$ minimal interpolation $[14,18]$. This idea was introduced and investigated in [14] for the Fourier-Padé approximations. The first step towards $L_{2}$-minimal interpolation was performed in [18]. The idea of this interpolation was in the determination of unknown parameters $\tau_{k}$ from the condition

$$
\lim _{N \rightarrow \infty} N^{q+1 / 2}\left\|r_{N, q}^{p}(f, x)\right\|_{L_{2}} \longrightarrow \text { minimum. }
$$

Paper [18] showed the solution of that problem for $p=1$ and $1 \leq q \leq 6$.

Another approach for the determination of parameters $\tau_{k}$ was described in [17], where $\tau_{k}$ were the roots of the associated Laguerre polynomial $L_{p}^{q}(x)$. Below, in numerical experiments, we use this approach.

The following theorem, proved in [15], shows the convergence rate of the rtp-interpolation in the regions away from the singularities $x= \pm 1$ for parameters $\theta_{k}$ chosen as in (32). 
Theorem 3 (see [15]). Let $f \in C^{q+2 p+1}[-1,1]$ and $f^{(q+2 p+1)} \in$ $A C[-1,1]$ for some $q, p \geq 1$. Let parameters $\theta_{k}$ be chosen as in (32). Then, the following estimate holds as $N \rightarrow \infty$ and $|x|<1$

$$
r_{N, q}^{p}(f, x)=O\left(N^{-q-2 p-1}\right)
$$

The main purpose of this paper is the derivation of the exact estimates of the $r_{N, q}^{p}(f, x)$ when $|x|<1$ getting more accurate ones than Theorem 3 presents. Based on such estimates, we discuss the problem of parameters $(\theta, p$, and $q$ ) determination for accurate interpolation. Theoretical estimates show the fast convergence of the rtp-interpolation compared to the KL-interpolation. Numerical experiments confirm theoretical estimates.

Throughout the paper, it is supposed that the exact values of the jumps $A_{k}(f)$ are known and that interpolated function is smooth on $[-1,1]$.

\section{Pointwise Convergence of the RTP-Interpolation}

Let $\theta_{k}$ be defined as in (32) and by $\gamma_{k}(\tau)$ denote the coefficients of the polynomial

$$
\prod_{s=1}^{p}\left(1+\tau_{s} x\right)=\sum_{s=0}^{p} \gamma_{s}(\tau) x^{s}
$$

Also denote

$\psi_{m, p}=\sum_{s=0}^{p}(-1)^{s} \gamma_{s}(\tau) \sum_{k=0}^{p} \gamma_{k}(\tau)(2 p-k-s+m) ! \phi_{2 p-k-s+m+1}$.

Theorems 4 and 5 describe pointwise behavior of the rtpinterpolation in the regions away from the singularities $x=$ \pm 1 . Theorem 4 deals with even values of $q$ and Theorem 5 with odd values.

Theorem 4. Let $q \geq 2$ be even and $f \in C^{q+2 p+1}[-1,1]$ with $f^{(q+2 p+1)} \in A C[-1,1]$ for some $p \geq 1$. Let parameters $\theta_{k}$ be chosen as in (32). Then, the following estimate holds for $|x|<1$,

$$
r_{N, q}^{p}(f, x)=\frac{\varphi_{N, q, p}(f, x)}{N^{2 p+q+1}}+o\left(N^{-2 p-q-1}\right), \quad N \longrightarrow \infty,
$$

where

$$
\varphi_{N, q, p}(f, x)=A_{q}(f) \frac{(-1)^{N+p+q / 2}}{2^{2 p+1} \pi^{q+1} q !} \frac{\sin (\pi(2 N+1) x / 2)}{\cos ^{2 p+1}(\pi x / 2)} \psi_{q, p} .
$$

Proof. Expansion (19) and definition of interpolation $I_{N, q}^{p}(f, x)$ show that

$$
r_{N, q}^{p}(f, x)=r_{N}^{p}(F, x)
$$

The application of transformation (14) to (17) with $\theta_{p+1}=1$ implies

$$
\begin{aligned}
r_{N, q}^{p}(f, x)= & \frac{\delta_{N}^{p}\left(\theta, \check{F}_{n}\right)}{c(x)}\left(e^{-i \pi N x}-e^{i \pi(N+1) x}\right) \\
& +\frac{\delta_{-N}^{p}\left(\theta, \check{F}_{n}\right)}{c(x)}\left(e^{i \pi N x}-e^{-i \pi(N+1) x}\right) \\
& +\frac{1}{c(x)} \sum_{n=-N}^{N} \delta_{n}^{1}\left(\delta_{n}^{p}\left(\theta, F_{n}-\check{F}_{n}\right)\right) e^{i \pi n x} \\
& +\frac{1}{c(x)} \sum_{|n|=N+1}^{\infty} \delta_{n}^{1}\left(\delta_{n}^{p}\left(\theta, F_{n}\right)\right) e^{i \pi n x},
\end{aligned}
$$

where, by $\delta_{n}^{s}\left(y_{n}\right)$, we denoted $\delta_{n}^{s}\left(\theta, y_{n}\right)$ with $\theta_{k}=1, k=$ $1, \ldots, s$ and

$$
\begin{aligned}
c(x) & =\left(1+e^{i \pi x}\right)\left(1+e^{-i \pi x}\right) \prod_{s=1}^{p}\left(1+\theta_{s} e^{i \pi x}\right)\left(1+\theta_{s} e^{-i \pi x}\right) \\
& =4 \cos ^{2} \frac{\pi x}{2} \prod_{s=1}^{p}\left(1+2 \theta_{s} \cos \pi x+\theta_{s}^{2}\right) .
\end{aligned}
$$

First, we estimate the last term in the right-hand side of (40). We need to estimate $\delta_{n}^{1}\left(\delta_{n}^{p}\left(\theta, F_{n}\right)\right)$ for $|n|>N$ as $N \rightarrow$ $\infty$. In view of the smoothness of $F$, we get from expansion (19) by means of integration by parts

$$
\begin{aligned}
F_{n}= & \sum_{m=q}^{q+2 p+1} A_{m}(f) B_{n}(m)+\frac{1}{2(i \pi n)^{2 p+q+2}} \\
& \times \int_{-1}^{1} f^{(2 p+q+2)}(x) e^{-i \pi n x} d x \\
= & \sum_{m=q}^{q+2 p+1} A_{m}(f) B_{n}(m)+o\left(n^{-2 p-q-2}\right),
\end{aligned}
$$

and consequently

$$
\begin{aligned}
\delta_{n}^{1}\left(\delta_{n}^{p}\left(\theta, F_{n}\right)\right)= & \sum_{m=q}^{q+2 p+1} A_{m}(f) \delta_{n}^{1}\left(\delta_{n}^{p}\left(\theta, B_{n}(m)\right)\right) \\
& +o\left(n^{-2 p-q-2}\right) .
\end{aligned}
$$

According to Lemma A.1,

$$
\delta_{n}^{1}\left(\delta_{n}^{p}\left(\theta, B_{n}(m)\right)\right)=\frac{1}{N^{2 p}} O\left(n^{-m-3}\right),
$$

and, therefore,

$$
\delta_{n}^{1}\left(\delta_{n}^{p}\left(\theta, F_{n}\right)\right)=\frac{1}{N^{2 p}} o\left(n^{-q-2}\right) .
$$

Thus, we conclude that the last term in the right-hand side of (40) is $o\left(N^{-q-2 p-1}\right)$. 
Now, we estimate the third term in the right-hand side of (40). We need to estimate $\delta_{n}^{1}\left(\delta_{n}^{p}\left(\theta, F_{n}-\breve{F}_{n}\right)\right)$ for $|n| \leq N$ as $N \rightarrow \infty$. Observing that

$$
\check{F}_{n}-F_{n}=\sum_{s \neq 0} F_{n+s(2 N+1)},
$$

and, applying (42), we obtain

$$
\begin{aligned}
& \check{F}_{n}-F_{n}= \sum_{m=q}^{q+2 p+1} A_{m}(f)\left(\sum_{s \neq 0} B_{n+s(2 N+1)}\right)+\frac{1}{N^{2 p+q+2}} \\
& \times \sum_{s \neq 0} \frac{\varepsilon_{n+s(2 N+1)}}{(n /(2 N+1)+s)^{q+2 p+2}} \\
&= \sum_{m=q}^{q+2 p+1} A_{m}(f)\left(\check{B}_{n}(m)-B_{n}(m)\right)+o\left(N^{-2 p-q-2}\right), \\
&|n| \leq N, \quad N \longrightarrow \infty,
\end{aligned}
$$

where, by $\varepsilon_{n}$, we denoted

$$
\varepsilon_{n}=\frac{1}{2(i \pi)^{2 p+q+2}} \int_{-1}^{1} f^{(2 p+q+2)}(x) e^{-i \pi n x} d x
$$

and took into account that $\varepsilon_{n}=o(1)$ as $n \rightarrow \infty$. Now, it follows that

$$
\begin{aligned}
\delta_{n}^{1}\left(\delta_{n}^{p}\left(\theta, \check{F}_{n}-F_{n}\right)\right) & \\
= & \sum_{m=q}^{q+2 p+1} A_{m}(f) \delta_{n}^{1}\left(\delta_{n}^{p}\left(\theta, \check{B}_{n}(m)-B_{n}(m)\right)\right) \\
& +o\left(N^{-2 p-q-2}\right) .
\end{aligned}
$$

According to Lemma A.2,

$$
\delta_{n}^{1}\left(\delta_{n}^{p}\left(\theta, \check{B}_{n}(m)-B_{n}(m)\right)\right)=O\left(N^{-2 p-q-3}\right),
$$

and, therefore,

$$
\delta_{n}^{1}\left(\delta_{n}^{p}\left(\theta, \check{F}_{n}-F_{n}\right)\right)=o\left(N^{-2 p-q-2}\right) .
$$

Hence, the third term in the right-hand side of (40) is also $o\left(N^{-q-2 p-1}\right)$ as $N \rightarrow \infty$.

Finally, we get

$$
\begin{aligned}
r_{N, q}^{p}(f, x)= & \frac{\delta_{N}^{p}\left(\theta, \check{F}_{n}\right)}{c(x)}\left(e^{-i \pi N x}-e^{i \pi(N+1) x}\right) \\
& +\frac{\delta_{-N}^{p}\left(\theta, \check{F}_{n}\right)}{c(x)}\left(e^{i \pi N x}-e^{-i \pi(N+1) x}\right) \\
& +o\left(N^{-q-2 p-1}\right),
\end{aligned}
$$

and we need to estimate $\delta_{ \pm N}^{p}\left(\theta, \check{F}_{n}\right)$. Similarly, as above,

$$
\delta_{ \pm N}^{p}\left(\theta, \check{F}_{n}\right)=\sum_{m=q}^{q+2 p+1} A_{m}(f) \delta_{ \pm N}^{p}\left(\theta, \check{B}_{n}(m)\right)+o\left(N^{-2 p-q-2}\right) .
$$

In view of Lemmas A.3 and A.4,

$$
\begin{aligned}
\delta_{ \pm N}^{p}\left(\theta, \check{F}_{n}\right)= & A_{q}(f) \delta_{ \pm N}^{p}\left(\theta, \check{B}_{n}(q)\right)+o\left(N^{-2 p-q-2}\right) \\
= & \pm A_{q}(f) \frac{(-1)^{N+p+1}}{2(i \pi)^{q+1} N^{2 p+q+1} q !} \psi_{q, p} \\
& +O\left(N^{-2 p-q-2}\right) .
\end{aligned}
$$

Substituting this into (52), we get the required estimate as $\theta_{k} \rightarrow 1$ and

$$
\lim _{N \rightarrow \infty} c(x)=2^{2 p+2} \cos ^{2 p+2} \frac{\pi x}{2} .
$$

We prove similar result for odd values of $q$.

Theorem 5. Let $q$ be odd, $q \geq 1$, and $f \in C^{q+2 p+2}[-1,1]$ with $f^{(q+2 p+2)} \in A C[-1,1]$ for some $p \geq 1$. Let parameters $\theta_{k}$ be chosen as in (32). Then, the following estimate holds for $|x|<1$,

$$
r_{N, q}^{p}(f, x)=\frac{\varphi_{N, q, p}(f, x)}{N^{2 p+q+2}}+o\left(N^{-2 p-q-2}\right), \quad N \longrightarrow \infty,
$$

where

$$
\begin{aligned}
\varphi_{N, q, p} & (f, x) \\
= & A_{q}(f) \frac{(-1)^{N+p+(q+1) / 2+1}}{2^{2 p+2} \pi^{q+1} q !} \\
& \times \frac{\sin (\pi x / 2) \sin (\pi(2 N+1) x / 2)}{\cos ^{2 p+2}(\pi x / 2)} \psi_{q+1, p} \\
& +A_{q+1}(f) \frac{(-1)^{N+p+(q+1) / 2}}{2^{2 p+1} \pi^{q+2}(q+1) !} \\
& \times \frac{\sin (\pi(2 N+1) x / 2)}{\cos ^{2 p+1}(\pi x / 2)} \psi_{q+1, p} .
\end{aligned}
$$

Proof. As the proof of this theorem mimics the proof of the previous one, we omit some details.

The application of transformation (14) to (17) twice with $\theta_{p+1}=\theta_{p+2}=1$ implies that

$$
\begin{aligned}
r_{N, q}^{p}(f, x)= & \frac{\delta_{N}^{p}\left(\theta, \check{F}_{n}\right)}{c(x)}\left(e^{-i \pi N x}-e^{i \pi(N+1) x}\right) \\
& +\frac{\delta_{-N}^{p}\left(\theta, \check{F}_{n}\right)}{c(x)}\left(e^{i \pi N x}-e^{-i \pi(N+1) x}\right) \\
& +\frac{\delta_{N}^{1}\left(\delta_{n}^{p}\left(\theta, \check{F}_{n}\right)\right)}{d(x)}\left(e^{-i \pi N x}-e^{i \pi(N+1) x}\right) \\
& +\frac{\delta_{-N}^{1}\left(\delta_{n}^{p}\left(\theta, \check{F}_{n}\right)\right)}{d(x)}\left(e^{i \pi N x}-e^{-i \pi(N+1) x}\right)
\end{aligned}
$$




$$
\begin{aligned}
& +\frac{1}{d(x)} \sum_{n=-N}^{N} \delta_{n}^{2}\left(\delta_{n}^{p}\left(\theta, F_{n}-\check{F}_{n}\right)\right) e^{i \pi n x} \\
& +\frac{1}{d(x)} \sum_{|n|=N+1}^{\infty} \delta_{n}^{2}\left(\delta_{n}^{p}\left(\theta, F_{n}\right)\right) e^{i \pi n x},
\end{aligned}
$$

where, by $\delta_{n}^{s}\left(y_{n}\right)$, we denoted $\delta_{n}^{s}\left(\theta, y_{n}\right)$ with $\theta_{k}=1, k=$ $1, \ldots, s$, and

$$
\begin{gathered}
c(x)=4 \cos ^{2} \frac{\pi x}{2} \prod_{s=1}^{p}\left(1+2 \theta_{s} \cos \pi x+\theta_{s}^{2}\right) \\
d(x)=\left(1+e^{i \pi x}\right)^{2}\left(1+e^{-i \pi x}\right)^{2} \prod_{s=1}^{p}\left(1+\theta_{s} e^{i \pi x}\right)\left(1+\theta_{s} e^{-i \pi x}\right) \\
=16 \cos ^{4} \frac{\pi x}{2} \prod_{s=1}^{p}\left(1+2 \theta_{s} \cos \pi x+\theta_{s}^{2}\right) .
\end{gathered}
$$

We have

$$
\begin{gathered}
F_{n}=\sum_{m=q}^{q+2 p+2} A_{m}(f) B_{n}(m)+o\left(n^{-2 p-q-3}\right), \\
\delta_{n}^{2}\left(\delta_{n}^{p}\left(\theta, F_{n}\right)\right)=\sum_{m=q}^{q+2 p+2} A_{m}(f) \delta_{n}^{2}\left(\delta_{n}^{p}\left(\theta, B_{n}(m)\right)\right) \\
+o\left(n^{-2 p-q-3}\right) .
\end{gathered}
$$

According to Lemma A.1,

$$
\delta_{n}^{2}\left(\delta_{n}^{p}\left(\theta, F_{n}\right)\right)=\frac{1}{N^{2 p}} o\left(n^{-q-3}\right),
$$

and the last term in the right-hand side of (58) is $o\left(N^{-q-2 p-2}\right)$ as $N \rightarrow \infty$.

Similarly,

$$
\begin{aligned}
& \delta_{n}^{2}\left(\delta_{n}^{p}\left(\theta, \check{F}_{n}-F_{n}\right)\right) \\
&=\sum_{m=q}^{q+2 p+2} A_{m}(f) \delta_{n}^{2}\left(\delta_{n}^{p}\left(\theta, \check{B}_{n}(m)-B_{n}(m)\right)\right) \\
& \quad+o\left(N^{-2 p-q-3}\right),
\end{aligned}
$$

and, according to Lemma A.2,

$$
\delta_{n}^{2}\left(\delta_{n}^{p}\left(\theta, \check{F}_{n}-F_{n}\right)\right)=o\left(N^{-2 p-q-3}\right), \quad N \longrightarrow \infty .
$$

Hence, the fifth term in the right-hand side of (58) is $o\left(N^{-q-2 p-2}\right)$ as $N \rightarrow \infty$.

Then,

$$
\begin{aligned}
\delta_{ \pm N}^{w}\left(\delta_{n}^{p}\left(\theta, \check{F}_{n}\right)\right)= & \sum_{m=q}^{q+2 p+2} A_{m}(f) \delta_{ \pm N}^{w}\left(\delta_{n}^{p}\left(\theta, \check{B}_{n}(m)\right)\right) \\
& +o\left(N^{-2 p-q-3}\right) .
\end{aligned}
$$

According to Lemma A.4, when $w=1$ in (64) and $q$ is odd, we get

$$
\begin{aligned}
\delta_{ \pm N}^{1}\left(\delta_{n}^{p}\left(\theta, \check{F}_{n}\right)\right)= & A_{q}(f) \delta_{ \pm N}^{1}\left(\delta_{n}^{p}\left(\theta, \check{B}_{n}(q)\right)\right) \\
& +o\left(N^{-2 p-q-3}\right) \\
= & o\left(N^{-2 p-q-3}\right),
\end{aligned}
$$

and hence the third and fourth terms in the right hand side of (58) are $o\left(N^{-q-2 p-2}\right)$ as $N \rightarrow \infty$. Now, from (58), we derive

$$
\begin{aligned}
r_{N, q}^{p}(f, x)= & \frac{\delta_{N}^{p}\left(\theta, \check{F}_{n}\right)}{c(x)}\left(e^{-i \pi N x}-e^{i \pi(N+1) x}\right) \\
& +\frac{\delta_{-N}^{p}\left(\theta, \check{F}_{n}\right)}{c(x)}\left(e^{i \pi N x}-e^{-i \pi(N+1) x}\right) \\
& +o\left(N^{-q-2 p-2}\right) .
\end{aligned}
$$

Taking $w=0$ in (64), we write

$$
\begin{aligned}
\delta_{ \pm N}^{p} & \left(\theta, \check{F}_{n}\right) \\
= & \sum_{m=q}^{q+2 p+2} A_{m}(f) \delta_{ \pm N}^{p}\left(\theta, \check{B}_{n}(m)\right)+o\left(N^{-2 p-q-3}\right) \\
= & A_{q}(f) \delta_{ \pm N}^{p}\left(\theta, \check{B}_{n}(q)\right)+A_{q+1}(f) \delta_{ \pm N}^{p}\left(\theta, \check{B}_{n}(q+1)\right) \\
& +o\left(N^{-2 p-q-3}\right) .
\end{aligned}
$$

Now, the application of Lemmas A.2 and A.3 completes the proof.

Note that Theorems 4 and 5 are valid also for $p=0$. In that case, the rtp-interpolation coincides with the KLinterpolation as $I_{N, q}^{0}(f, x) \equiv I_{N, q}(f, x)$ and consequently $r_{N, q}^{0}(f, x) \equiv r_{N, q}(f, x)$. Hence, for $p=0$, Theorems 4 and 5 coincide with Theorems 1 and 2, respectively.

\section{Results and Discussion}

First, let us compare Theorems 4 and 5. Theorem 4 states that on the interval $|x|<1$, the rate of convergence of $I_{N, q}^{p}(f, x)$ is $O\left(N^{-q-2 p-1}\right)$ for even values of $q$. According to Theorem 5, the rate of convergence of $I_{N, q}^{p}(f, x)$ for odd values of $q$ is $O\left(N^{-q-2 p-2}\right)$, and we have an improvement in the convergence by the factor $O(N)$. From the other side, Theorem 5 puts an additional smoothness requirement on the interpolated function. Moreover, while the estimate in Theorem 4 depends only on $A_{q}(f)$, the estimate of Theorem 5 depends on both $A_{q}(f)$ and $A_{q+1}(f)$. All these mimic the behavior of the KL-interpolation where we have the same differences in the asymptotic estimates of Theorems 1 and 2.

Now, let us compare convergence of the KL- and the rtpinterpolations. In this section, we suppose that parameters 
$\tau_{k}$ in (32) are the roots of the associated Laguerre polynomial $L_{p}^{q}(x)$. Theorems concerning the rtp-interpolations put additional smoothness requirements on the interpolated function, and, in comparisons, it must be taken into account.

If an interpolated function is rather smooth (e.g., when function is infinitely differentiable) such that for given $q$ and $p$ Theorems 4 and 5 are valid, then the rtp-interpolation is more precise (however, asymptotically) than the KL-interpolation. Comparison of Theorems 1 and 2 with Theorems 4 and 5 shows that the rtp-interpolation is more precise than the KLinterpolation by factor $O\left(N^{2 p}\right)$.

Now, we consider functions with finite smoothness and suppose that all needed jumps are exactly available. Estimates proved above show (see factors $A_{q}(f)$ and $A_{q+1}(f)$ ) that the utilization of all available jumps by the KL-interpolation is not reasonable when the jumps of the interpolated function are rapidly increasing. In such cases, more accuracy can be achieved with less jumps in combination with rational corrections.

Further in this section, we suppose that parameters $\tau_{k}$ are the roots of the associated Laguerre polynomials $L_{p}^{q}(x)$.

Let $f \in C^{M+1}[-1,1], f^{(M+1)} \in A C[-1,1], M \geq 1$, and let $q$ be an even number. According to Theorems 1 and 4, if the values of $p$ and $q$ satisfy the condition $q+2 p=M$, then both theorems are valid, and comparison of corresponding approximations is legal. Then, asymptotic estimates of these theorems will show which values of parameters $p$ and $q$ provide with better accuracy. We show this process for a specific example. Let

$$
f(x)=\sin (a x-1),
$$

where $a$ is some parameter. We use the values $a=$ $1 / 10,1,10,30,50$, and in Table 1 to calculate the values of $N^{-q-2 p-1} \max _{x \in[-0.5,0.5]}\left|\varphi_{N, q, p}(f, x)\right|$ for $N=1024$ and for different values of $p$ and $q$ with condition $q+2 p=8$. Recall that $p=0$ corresponds to the KL-interpolation.

Table 1 shows that for $a=1 / 10$ and $a=1$ utilization of all available jumps is reasonable and the KL-interpolation $I_{N, 8}(f, x)$ has the best accuracy. For $a=10,30$, and 50, the rtp-interpolation $I_{N, 2}^{3}(f, x)$ is the best, and as larger is the value of parameter $a$ as more precise is the rtp-interpolation compared to the KL-interpolation.

It is important to note that results in Table 1 are obtained based on asymptotic estimates and we must compare these results to actual errors $\max _{x \in[-0.5,0.5]}\left|r_{N, q}^{p}(f, x)\right|$ to see how the asymptotic errors coincide with actual errors. In Table 2 , the values of the actual errors $\max _{x \in[-0.5,0.5]}\left|r_{N, q}^{p}(f, x)\right|$ for $N=$ 1024 , and for different values of $p$ and $q$ with condition $q+$ $2 p=8$ are calculated. Comparison with Table 1 shows that for $N=1024$ theoretical estimates of Theorems 1 and 4 coincide with actual errors rather precisely.

We have the same situation for odd values of $q$. Let $f \in$ $C^{M+2}[-1,1], f^{(M+2)} \in A C[-1,1], M \geq 1$, and let $q$ be an odd number. We use the same values of $a$ and in Table 3 to calculate the values of $\left(1 / N^{q+2 p+2}\right) \max _{x \in[-0.5,0.5]}\left|\varphi_{N, q, p}(f, x)\right|$ and in Table 4 to calculate the values of $\max _{x \in[-0.5,0.5]}\left|r_{N, q}^{p}(f)\right|$ for $N=1024$ and for different values of $p$ and $q$ with
TABLE 1: Values of $N^{-q-2 p-1} \max _{x \in[-0.5,0.5]}\left|\varphi_{N, q, p}(f, x)\right|$ (see (38)) for $N=1024, q+2 p=8$ and for function (68). Parameters $\tau_{k}$ are the roots of the associated Laguerre polynomials $L_{p}^{q}(x)$.

\begin{tabular}{lcccc}
\hline & $p=3$ & $p=2$ & $p=1$ & $p=0$ \\
& $q=2$ & $q=4$ & $q=6$ & $q=8$ \\
\hline$a=1 / 10$ & $1.5 \cdot 10^{-30}$ & $4.6 \cdot 10^{-34}$ & $1.2 \cdot 10^{-37}$ & $3.9 \cdot 10^{-41}$ \\
$a=1$ & $1.2 \cdot 10^{-27}$ & $3.9 \cdot 10^{-29}$ & $1.1 \cdot 10^{-30}$ & $3.3 \cdot 10^{-32}$ \\
$a=10$ & $8.0 \cdot 10^{-26}$ & $2.5 \cdot 10^{-25}$ & $6.8 \cdot 10^{-25}$ & $2.2 \cdot 10^{-24}$ \\
$a=30$ & $1.3 \cdot 10^{-24}$ & $3.7 \cdot 10^{-23}$ & $9.0 \cdot 10^{-22}$ & $2.6 \cdot 10^{-22}$ \\
$a=50$ & $9.7 \cdot 10^{-25}$ & $7.6 \cdot 10^{-23}$ & $5.1 \cdot 10^{-21}$ & $4.1 \cdot 10^{-19}$ \\
\hline
\end{tabular}

TABLE 2: Values of $\max _{x \in[-0.5,0.5]}\left|r_{N, q}^{p}(f, x)\right|$ (see (26)) for $N=1024$, $q+2 p=8$, and for function (68). Parameters $\tau_{k}$ are the roots of the associated Laguerre polynomials $L_{p}^{q}(x)$.

\begin{tabular}{lcccc}
\hline & $p=3$ & $p=2$ & $p=1$ & $p=0$ \\
& $q=2$ & $q=4$ & $q=6$ & $q=8$ \\
\hline$a=1 / 10$ & $1.2 \cdot 10^{-30}$ & $3.9 \cdot 10^{-34}$ & $1.2 \cdot 10^{-37}$ & $3.9 \cdot 10^{-41}$ \\
$a=1$ & $9.8 \cdot 10^{-28}$ & $3.3 \cdot 10^{-29}$ & $9.9 \cdot 10^{-31}$ & $3.3 \cdot 10^{-32}$ \\
$a=10$ & $6.3 \cdot 10^{-26}$ & $2.1 \cdot 10^{-25}$ & $6.4 \cdot 10^{-25}$ & $2.1 \cdot 10^{-24}$ \\
$a=30$ & $1.0 \cdot 10^{-24}$ & $3.1 \cdot 10^{-23}$ & $8.5 \cdot 10^{-22}$ & $2.6 \cdot 10^{-22}$ \\
$a=50$ & $7.8 \cdot 10^{-25}$ & $6.4 \cdot 10^{-23}$ & $4.8 \cdot 10^{-21}$ & $4.0 \cdot 10^{-19}$ \\
\hline
\end{tabular}

TABLE 3: Values of $N^{-2 p-q-2} \max _{x \in[-0.5,0.5]}\left|\varphi_{N, q, p}(f, x)\right|$ (see (57)) for $N=1024, q+2 p=7$ and for function (68). Parameters $\tau_{k}$ are the roots of the associated Laguerre polynomials $L_{p}^{q}(x)$.

\begin{tabular}{lcccc}
\hline & $p=3$ & $p=2$ & $p=1$ & $p=0$ \\
& $q=1$ & $q=3$ & $q=5$ & $q=7$ \\
\hline$a=1 / 10$ & $4.0 \cdot 10^{-28}$ & $2.0 \cdot 10^{-31}$ & $4.8 \cdot 10^{-35}$ & $7.1 \cdot 10^{-39}$ \\
$a=1$ & $4.0 \cdot 10^{-26}$ & $1.9 \cdot 10^{-27}$ & $4.3 \cdot 10^{-29}$ & $6.3 \cdot 10^{-31}$ \\
$a=10$ & $6.6 \cdot 10^{-25}$ & $2.3 \cdot 10^{-24}$ & $4.5 \cdot 10^{-24}$ & $6.0 \cdot 10^{-24}$ \\
$a=30$ & $8.6 \cdot 10^{-24}$ & $2.3 \cdot 10^{-22}$ & $3.7 \cdot 10^{-21}$ & $4.1 \cdot 10^{-20}$ \\
$a=50$ & $6.0 \cdot 10^{-24}$ & $4.3 \cdot 10^{-22}$ & $1.9 \cdot 10^{-20}$ & $5.5 \cdot 10^{-19}$ \\
\hline
\end{tabular}

TABLE 4: Values of $\max _{x \in[-0.5,0.5]}\left|r_{N, q}^{p}(f, x)\right|$ (see (26)) for $N=1024$, $q+2 p=7$ and for function (68). Parameters $\tau_{k}$ are the roots of the associated Laguerre polynomials $L_{p}^{q}(x)$.

\begin{tabular}{lcccc}
\hline & $p=3$ & $p=2$ & $p=1$ & $p=0$ \\
& $q=1$ & $q=3$ & $q=5$ & $q=7$ \\
\hline$a=1 / 10$ & $3.8 \cdot 10^{-28}$ & $2.0 \cdot 10^{-31}$ & $4.7 \cdot 10^{-35}$ & $7.0 \cdot 10^{-39}$ \\
$a=1$ & $3.8 \cdot 10^{-26}$ & $1.8 \cdot 10^{-27}$ & $4.2 \cdot 10^{-29}$ & $6.2 \cdot 10^{-31}$ \\
$a=10$ & $6.3 \cdot 10^{-25}$ & $2.2 \cdot 10^{-24}$ & $4.5 \cdot 10^{-24}$ & $6.0 \cdot 10^{-24}$ \\
$a=30$ & $8.1 \cdot 10^{-24}$ & $2.3 \cdot 10^{-22}$ & $3.7 \cdot 10^{-21}$ & $4.1 \cdot 10^{-20}$ \\
$a=50$ & $5.7 \cdot 10^{-24}$ & $4.2 \cdot 10^{-22}$ & $1.8 \cdot 10^{-20}$ & $5.5 \cdot 10^{-19}$ \\
\hline
\end{tabular}

condition $q+2 p=7$ when both Theorems 2 and 5 are valid. Again, for $a=1 / 10$ and $a=1$, the best accuracy has the KLinterpolation. For $a=10, a=30$, and $a=50$, the best is rtp-interpolation $I_{N, 1}^{3}(f, x)$.

Overall conclusion based on these specific examples and on comparison of the asymptotic estimates is the following: 
not always the utilization of all available jumps, by the KLinterpolation, leads to the best interpolation (if we mean pointwise convergence in the regions away from the singularities where the rtp-interpolation has faster convergence rate.) This is due to the factors $A_{q}(f)$ and $A_{q+1}(f)$ in the estimates. When the values of jumps are rapidly increasing, then better accuracy can be achieved by the utilization of smaller number of jumps (consequently, with smaller $A_{q}(f)$ and $\left.A_{q+1}(f)\right)$ and appropriately chosen corrections based on the smoothness of the interpolated function. Which choice of $q$ and $p$ is the best that can be concluded from the comparison of the corresponding estimates as we did it above.

It must be also mentioned that when the jumps are rapidly increasing then getting their approximations is problematic, so in such cases, the utilization of the rational corrections is unavoidable for better accuracy.

\section{Appendix}

In this section, we prove some lemmas concerning generalized finite differences $\delta_{n}^{s}\left(\theta, y_{n}\right)$. By $\delta_{n}^{s}\left(y_{n}\right)$, we denote the sequence that corresponds to $\theta_{k}=1, k=1, \ldots, s$. By $\Delta_{n}^{k}\left(c_{n}\right)$, we denote the differences defined by the relations

$$
\begin{gathered}
\Delta_{n}^{0}\left(c_{n}\right)=c_{n}, \\
\Delta_{n}^{k}\left(c_{n}\right)=\Delta_{n}^{k-1}\left(c_{n}\right)+\Delta_{n-1}^{k-1}\left(c_{n}\right) .
\end{gathered}
$$

Now, it is easy to verify that

$$
\delta_{n}^{k}\left(c_{n}\right)=\Delta_{n+k}^{2 k}\left(c_{n}\right) .
$$

Lemma A.1. The following estimate holds for $p, w, m \geq 0$ as $N \rightarrow \infty$ and $|n| \geq N+1$, where $\theta_{k}$ are chosen as in (32)

$$
\begin{aligned}
\delta_{n}^{w}\left(\delta_{n}^{p}\left(\theta, B_{n}(m)\right)\right)= & \frac{(-1)^{n+p+w+1}}{2(i \pi n)^{m+1} n^{2 w+2 p} m !} \\
& \times \sum_{s=0}^{p}(-1)^{s} \frac{\gamma_{s}(\tau)}{N^{s} n^{-s}} \sum_{k=0}^{p} \frac{\gamma_{k}(\tau)}{N^{k} n^{-k}} \\
& \times(2 w+2 p-k-s+m) ! \\
& +\frac{1}{N^{2 p}} O\left(n^{-2 w-m-2}\right) .
\end{aligned}
$$

Proof. In view of definitions of $\delta_{n}^{k}\left(\theta, y_{n}\right), \delta_{n}^{k}\left(y_{n}\right)$ and their connection with $\Delta_{n}^{k}\left(y_{n}\right)$, we write

$$
\begin{aligned}
\delta_{n}^{p} & \left(\theta, B_{n}(m)\right) \\
& =\sum_{s=0}^{p}(-1)^{s} \frac{\gamma_{s}(\tau)}{N^{s}} \sum_{k=0}^{p}(-1)^{k} \frac{\gamma_{k}(\tau)}{N^{k}} \Delta_{n+p-s}^{2 p-k-s}\left(B_{n}(m)\right) .
\end{aligned}
$$

Then,

$$
\begin{aligned}
\delta_{n}^{w} & \left(\delta_{n}^{p}\left(\theta, B_{n}(m)\right)\right) \\
& =\sum_{s=0}^{p}(-1)^{s} \frac{\gamma_{s}(\tau)}{N^{s}} \sum_{k=0}^{p}(-1)^{k} \frac{\gamma_{k}(\tau)}{N^{k}} \Delta_{n+w}^{2 w}\left(\Delta_{n+p-s}^{2 p-k-s}\left(B_{n}(m)\right)\right) .
\end{aligned}
$$

Taking into account that

$$
\Delta_{n+w}^{2 w}\left(\Delta_{n+p-s}^{2 p-k-s}\left(B_{n}(m)\right)\right)=\Delta_{n+p-s+w}^{2 w+2 p-k-s}\left(B_{n}(m)\right),
$$

we find

$$
\begin{aligned}
\delta_{n}^{w} & \left(\delta_{n}^{p}\left(\theta, B_{n}(m)\right)\right) \\
& =\sum_{s=0}^{p}(-1)^{s} \frac{\gamma_{s}(\tau)}{N^{s}} \sum_{k=0}^{p}(-1)^{k} \frac{\gamma_{k}(\tau)}{N^{k}} \Delta_{n+p-s+w}^{2 w+2 p-k-s}\left(B_{n}(m)\right) .
\end{aligned}
$$

Moreover, since

$$
\Delta_{n}^{w}\left(y_{n}\right)=\sum_{\ell=0}^{2 w}\left(\begin{array}{c}
2 w \\
\ell
\end{array}\right) y_{n-\ell},
$$

we obtain

$$
\begin{aligned}
\delta_{n}^{w}\left(\delta_{n}^{p}\left(\theta, B_{n}(m)\right)\right) \\
=\sum_{s=0}^{p}(-1)^{s} \frac{\gamma_{s}(\tau)}{N^{s}} \sum_{k=0}^{p}(-1)^{k} \frac{\gamma_{k}(\tau)}{N^{k}} \\
\quad \times \sum_{\ell=0}^{2 w+2 p-k-s}\left(\begin{array}{c}
2 w+2 p-k-s \\
\ell
\end{array}\right) B_{n+p-s+w-\ell}(m) .
\end{aligned}
$$

Taking into account the explicit form of the Fourier coefficients of the Bernoulli polynomials

$$
\begin{aligned}
B_{n+p-s+w-\ell}(m)= & \frac{(-1)^{n+p-s+w-\ell+1}}{2(i \pi(n+p-s+w-\ell))^{m+1}} \\
= & \frac{(-1)^{n+p+s+w+\ell+1}}{2(i \pi n)^{m+1}} \\
& \times \sum_{j=0}^{\infty}\left(\begin{array}{c}
j+m \\
m
\end{array}\right) \frac{(\ell+s-w-p)^{j}}{n^{j}} \\
= & \frac{(-1)^{n+p+s+w+\ell+1}}{2(i \pi n)^{m+1}} \sum_{j=0}^{\infty} \frac{1}{n^{j}}\left(\begin{array}{c}
j+m \\
m
\end{array}\right) \\
& \times \sum_{t=0}^{j}\left(\begin{array}{c}
j \\
t
\end{array}\right)^{t}(s-w-p)^{j-t},
\end{aligned}
$$

we derive

$$
\begin{aligned}
\delta_{n}^{w}\left(\delta_{n}^{p}\left(\theta, B_{n}(m)\right)\right) \\
=\frac{(-1)^{n+\mathrm{p}+w+1}}{2(i \pi n)^{m+1}} \sum_{s=0}^{p} \frac{\gamma_{s}(\tau)}{N^{s}} \sum_{k=0}^{p}(-1)^{k} \frac{\gamma_{k}(\tau)}{N^{k}} \\
\quad \times \sum_{j=0}^{\infty} \frac{1}{n^{j}}\left(\begin{array}{c}
j+m \\
m
\end{array}\right) \\
\quad \times \sum_{t=0}^{j}\left(\begin{array}{c}
j \\
t
\end{array}\right)(s-w-p)^{j-t} \alpha_{2 w+2 p-k-s, t},
\end{aligned}
$$


where

$$
\alpha_{r, t}=\sum_{\ell=0}^{r}(-1)^{\ell}\left(\begin{array}{l}
r \\
\ell
\end{array}\right) \ell^{t} .
$$

Note that

$$
\alpha_{r, t}=0, \quad t \leq r-1 \text {. }
$$

Thus,

$$
\begin{aligned}
& \delta_{n}^{w}\left(\delta_{n}^{p}\left(\theta, B_{n}(m)\right)\right) \\
&=\frac{(-1)^{n+p+w+1}}{2(i \pi n)^{m+1} n^{2 w+2 p}} \sum_{s=0}^{p} \frac{\gamma_{s}(\tau)}{N^{s}} \sum_{k=0}^{p}(-1)^{k} \frac{\gamma_{k}(\tau)}{N^{k}} \\
& \times \sum_{j=0}^{\infty} \frac{1}{n^{j-k-s}}\left(\begin{array}{c}
2 w+2 p-k-s+j+m \\
m
\end{array}\right) \\
& \times \sum_{t=0}^{j}\left(\begin{array}{l}
j+2 w+2 p-k-s \\
t+2 w+2 p-k-s
\end{array}\right)(s-w-p)^{j-t} \\
& \times \alpha_{2 w+2 p-k-s, t+2 w+2 p-k-s} \\
&= \frac{(-1)^{n+p+w+1}}{2(i \pi n)^{m+1} n^{2 w+2 p} m !} \sum_{s=0}^{p} \frac{\gamma_{s}(\tau)}{N^{s} n^{-s}} \sum_{k=0}^{p}(-1)^{k} \frac{\gamma_{k}(\tau)}{N^{k} n^{-k}} \\
& \times\left(\begin{array}{c}
2 w+2 p-k-s+m \\
m
\end{array}\right) \alpha_{2 w+2 p-k-s, 2 w+2 p-k-s} \\
&+\frac{1}{N^{2 p}} O\left(n^{-2 w-m-2}\right),
\end{aligned}
$$

which completes the proof as

$$
\alpha_{2 w+2 p-k-s, 2 w+2 p-k-s}=(-1)^{2 w+2 p-k-s}(2 w+2 p-k-s) ! .
$$

The proof of next lemmas can be obtained in a similar manner.

Lemma A.2. The following estimate holds for $p, w, m \geq 0$ as $N \rightarrow \infty$ and $|n| \leq N$, where $\theta_{k}$ are chosen as in (32)

$$
\begin{aligned}
\delta_{n}^{w}\left(\delta_{n}^{p}\right. & \left.\left(\theta, \check{B}_{n}(m)-B_{n}(m)\right)\right) \\
= & \frac{(-1)^{n+p+w+1}}{2(i \pi N)^{m+1} N^{2 w+2} p m !} \sum_{s=0}^{p}(-1)^{s} \gamma_{s}(\tau) \sum_{k=0}^{p} \gamma_{k}(\tau) \\
& \times(2 w+2 p-k-s+m) ! \\
& \times \sum_{r \neq 0} \frac{(-1)^{r}}{(2 r+n / N)^{2 w+2 p-k-s+m+1}} \\
& +O\left(N^{-2 w-2 p-m-2}\right) .
\end{aligned}
$$

Proof. Similar to (A.9), we get

$$
\begin{aligned}
\delta_{n}^{w}\left(\delta_{n}^{p}\left(\theta, \check{B}_{n}(m)-B_{n}(m)\right)\right) & \\
= & \sum_{s=0}^{p}(-1)^{s} \frac{\gamma_{s}(\tau)}{N^{s}} \sum_{k=0}^{p}(-1)^{k} \frac{\gamma_{k}(\tau)}{N^{k}} \\
& \times \sum_{\ell=0}^{2 w+2 p-k-s}\left(\begin{array}{c}
2 w+2 p-k-s \\
\ell
\end{array}\right) \\
& \times\left(\check{B}_{n+p-s+w-\ell}(m)-B_{n+p-s+w-\ell}(m)\right) .
\end{aligned}
$$

Taking into account that

$$
\begin{aligned}
\check{B}_{n}(m)-B_{n}(m) & =\sum_{r \neq 0} B_{n+r(2 N+1)}(m) \\
& =\frac{(-1)^{n+1}}{2(i \pi)^{m+1}} \sum_{r \neq 0} \frac{(-1)^{r}}{(n+r(2 N+1))^{m+1}},
\end{aligned}
$$

we derive

$$
\begin{aligned}
\delta_{n}^{w}\left(\delta_{n}^{p}\right. & \left.\left(\theta, \check{B}_{n}(m)-B_{n}(m)\right)\right) \\
= & \frac{(-1)^{n+p+w+1}}{2(i \pi N)^{m+1}} \sum_{s=0}^{p} \frac{\gamma_{s}(\tau)}{N^{s}} \sum_{k=0}^{p}(-1)^{k} \frac{\gamma_{k}(\tau)}{N^{\mathrm{k}}} \\
& \times \sum_{j=0}^{\infty} \frac{1}{N^{j}}\left(\begin{array}{c}
j+m \\
m
\end{array}\right) \sum_{t=0}^{j}\left(\begin{array}{c}
j \\
t
\end{array}\right) \alpha_{2 w+2 p-k-s, t} \\
& \times \sum_{r \neq 0} \frac{(-1)^{r}(s-w-p-r)^{j-t}}{(2 r+n / N)^{j+m+1}} .
\end{aligned}
$$

In view of identity (A.13), we have

$$
\begin{aligned}
\delta_{n}^{w}( & \left.\delta_{n}^{p}\left(\theta, \check{B}_{n}(m)-B_{n}(m)\right)\right) \\
= & \frac{(-1)^{n+p+w+1}}{2(i \pi N)^{m+1} N^{2 w+2 p}} \sum_{s=0}^{p} \gamma_{s}(\tau) \sum_{k=0}^{p}(-1)^{k} \gamma_{k}(\tau) \\
& \times \sum_{j=0}^{\infty} \frac{1}{N^{j}}\left(\begin{array}{c}
j+2 w+2 p-k-s+m \\
m
\end{array}\right) \\
& \times \sum_{t=0}^{j}\left(\begin{array}{l}
j+2 w+2 p-k-s \\
t+2 w+2 p-k-s
\end{array}\right) \\
& \times \alpha_{2 w+2 p-k-s, t+2 w+2 p-k-s} \sum_{r \neq 0} \frac{(-1)^{r}(s-w-p-r)^{j-t}}{(2 r+n / N)^{j+2 w+2 p-k-s+m+1}}
\end{aligned}
$$




$$
\begin{aligned}
= & \frac{(-1)^{n+p+w+1}}{2(i \pi N)^{m+1} N^{2 w+2 p}} \\
& \times \sum_{s=0}^{p} \gamma_{s}(\tau) \sum_{k=0}^{p}(-1)^{k} \gamma_{k}(\tau)\left(\begin{array}{c}
2 w+2 p-k-s+m \\
m
\end{array}\right) \\
& \times \alpha_{2 w+2 p-k-s, 2 w+2 p-k-s} \\
& \times \sum_{r \neq 0} \frac{(-1)^{r}}{(2 r+n / N)^{2 w+2 p-k-s+m+1}}+O\left(N^{-2 w-2 p-m-2}\right),
\end{aligned}
$$

which completes the proof as

$$
\alpha_{2 w+2 p-k-s, 2 w+2 p-k-s}=(-1)^{2 w+2 p+k+s}(2 w+2 p-k-s) ! .
$$

Lemma A.3. Let $m$ be even. Then, the following estimate holds for $p, w, m \geq 0$ as $N \rightarrow \infty$, where $\theta_{k}$ are chosen as in (32)

$$
\begin{aligned}
\delta_{ \pm N}^{w} & \left(\delta_{n}^{p}\left(\theta, \check{B}_{n}(m)\right)\right) \\
= & \frac{(-1)^{N+p+w+1}}{2(i \pi N)^{m+1} N^{2 w+2 p} m !} \sum_{s=0}^{p}(-1)^{s} \gamma_{s}(\tau) \sum_{k=0}^{p} \gamma_{k}(\tau) \\
& \times(2 w+2 p-k-s+m) ! \sum_{r=-\infty}^{\infty} \frac{(-1)^{r}}{(2 r \pm 1)^{2 w+2 p-k-s+m+1}} \\
& +O\left(N^{-2 w-2 p-m-2}\right) .
\end{aligned}
$$

Proof. Similar to (A.9), we get

$$
\begin{aligned}
\delta_{ \pm N}^{w}( & \left.\delta_{n}^{p}\left(\theta, \check{B}_{n}(m)\right)\right) \\
= & \sum_{s=0}^{p}(-1)^{s} \frac{\gamma_{s}(\tau)}{N^{s}} \sum_{k=0}^{p}(-1)^{k} \frac{\gamma_{k}(\tau)}{N^{k}} \\
& \times \sum_{\ell=0}^{2 w+2 p-k-s}\left(\begin{array}{c}
2 w+2 p-k-s \\
\ell
\end{array}\right) \check{B}_{ \pm N+p-s+w-\ell}(m) .
\end{aligned}
$$

Taking into account that

$$
\begin{aligned}
\check{B}_{n}(m) & =\sum_{s=-\infty}^{\infty} B_{n+s(2 N+1)}(m) \\
& =\frac{(-1)^{n+1}}{2(i \pi)^{m+1}} \sum_{s=-\infty}^{\infty} \frac{(-1)^{s}}{(n+s(2 N+1))^{m+1}},
\end{aligned}
$$

we have

$$
\begin{aligned}
\check{B}_{ \pm N+p-s+w-\ell}(m)= & \frac{(-1)^{N+p+w+s+\ell+1}}{2(i \pi N)^{m+1}} \sum_{j=0}^{\infty} \frac{1}{N^{j}}\left(\begin{array}{c}
j+m \\
m
\end{array}\right) \\
& \times \sum_{r=-\infty}^{\infty} \frac{(-1)^{r}(\ell+s-p-w-r)^{j}}{(2 r \pm 1)^{m+j+1}} .
\end{aligned}
$$

Hence,

$$
\begin{aligned}
\delta_{ \pm N}^{w}\left(\delta_{n}^{p}\left(\theta, \check{B}_{n}(m)\right)\right) \\
=\frac{(-1)^{N+p+w+1}}{2(i \pi N)^{m+1} N^{2 w+2 p}} \sum_{s=0}^{p} \gamma_{s}(\tau) \sum_{k=0}^{p}(-1)^{k} \gamma_{k}(\tau) \\
\quad \times \sum_{j=0}^{\infty} \frac{1}{N^{j}}\left(\begin{array}{c}
j+2 w+2 p-k-s+m \\
m
\end{array}\right) \\
\quad \times \sum_{t=0}^{j}\left(\begin{array}{l}
j+2 w+2 p-k-s \\
t+2 w+2 p-k-s
\end{array}\right) \alpha_{2 w+2 p-k-s, t+2 w+2 p-k-s} \\
\quad \times \sum_{r=-\infty}^{\infty} \frac{(-1)^{r}(s-p-w-r)^{j-t}}{(2 r \pm 1)^{j+2 w+2 p-k-s+m+1}} .
\end{aligned}
$$

From here, we get

$$
\begin{aligned}
\delta_{ \pm N}^{w} & \left(\delta_{n}^{p}\left(\theta, \check{B}_{n}(m)\right)\right) \\
= & \frac{(-1)^{N+p+w+1}}{2(i \pi N)^{m+1} N^{2 w+2 p}} \sum_{s=0}^{p} \gamma_{s}(\tau) \sum_{k=0}^{p}(-1)^{k} \gamma_{k}(\tau) \\
& \times\left(\begin{array}{c}
2 w+2 p-k-s+m \\
m
\end{array}\right) \alpha_{2 w+2 p-k-s, 2 w+2 p-k-s} \\
& \times \sum_{r=-\infty}^{\infty} \frac{(-1)^{r}}{(2 r \pm 1)^{2 w+2 p-k-s+m+1}}+O\left(N^{-2 w-2 p-m-2}\right),
\end{aligned}
$$

which completes the proof.

Lemma A.4. Let $m$ be odd. Then, the following estimate holds for $p, w \geq 0$ and $m \geq 1$ as $N \rightarrow \infty$, where $\theta_{k}$ are chosen as in (32)

$$
\begin{aligned}
\delta_{ \pm N}^{w} & \left(\delta_{n}^{p}\left(\theta, \check{B}_{n}(m)\right)\right) \\
= & \frac{(-1)^{N+p+w}}{2(i \pi N)^{m+1} N^{2 w+2 p+1} m !} \sum_{s=0}^{p}(-1)^{s} \gamma_{s}(\tau) \sum_{k=0}^{p} \gamma_{k}(\tau) \\
& \times(2 w+2 p-k-s+m+1) ! \\
& \times \sum_{r=-\infty}^{\infty} \frac{(-1)^{r} r}{(2 r \pm 1)^{2 w+2 p-k-s+m+2}} \\
& +O\left(N^{-2 w-2 p-m-3}\right) .
\end{aligned}
$$


Proof. We again use (A.26) and explore terms $j=0$ and $j=1$

$$
\begin{aligned}
\delta_{ \pm N}^{w}( & \left.\delta_{n}^{p}\left(\theta, \check{B}_{n}(m)\right)\right) \\
= & \frac{(-1)^{N+p+w+1}}{2(i \pi N)^{m+1} N^{2 w+2 p} m !} \sum_{s=0}^{p}(-1)^{s} \gamma_{s}(\tau) \sum_{k=0}^{p} \gamma_{k}(\tau) \\
& \times(2 w+2 p-k-s+m) ! \sum_{r=-\infty}^{\infty} \frac{(-1)^{r}}{(2 r \pm 1)^{2 w+2 p-k-s+m+1}} \\
& +\frac{(-1)^{N+p+w+1}}{4(i \pi N)^{m+1} N^{2 w+2 p+1} m !} \sum_{s=0}^{p}(-1)^{s} \gamma_{s}(\tau) \sum_{k=0}^{p} \gamma_{k}(\tau) \\
& \times(2 w+2 p-k-s+m+1) !(s-k) \\
& \times \sum_{r=-\infty}^{\infty} \frac{(-1)^{r}}{(2 r \pm 1)^{2 w+2 p-k-s+m+2}} \\
& +\frac{(-1)^{N+p+w}}{2(i \pi N)^{m+1} N^{2 w+2 p+1} m !} \sum_{s=0}^{p}(-1)^{s} \gamma_{s}(\tau) \sum_{k=0}^{p} \gamma_{k}(\tau) \\
& \times(2 w+2 p-k-s+m+1) ! \\
& \times \sum_{r=-\infty}^{\infty} \frac{(-1)^{r} r}{(2 r \pm 1)^{2 w+2 p-k-s+m+2}} \\
& \left.+O(N)^{-2 w-2 p-m-3}\right), \quad N-\infty
\end{aligned}
$$

It is easy to verify that the first two terms in the right-hand side of (A.29) vanish for odd values of $m$. This observation completes the proof.

\section{Notations}

$f_{n}: \quad$ The Fourier coefficient (see (5))

$\check{f}_{n}: \quad$ The discrete Fourier coefficient (see (2))

$I_{N}(f, x)$ : Classic trigonometric interpolation (see (1))

$r_{N}(f, x)$ : Error of classic trigonometric interpolation (see (4))

$I_{N}^{p}(f, x)$ : Rational-trigonometric interpolation (see (16))

$r_{N}^{p}(f, x): \quad$ Error of rational-trigonometric interpolation (see (17))

$I_{N, q}(f, x)$ : The Krylov-Lanczos interpolation (see (23))

$r_{N, q}(f, x)$ : Error of the Krylov-Lanczos interpolation (see (25))

$I_{N, q}^{p}(f, x)$ : Rational-trigonometric-polynomial interpolation (see (24))

$r_{N, q}^{p}(f, x):$ Error of rational-trigonometric-polynomial interpolation (see (26))

$A_{k}(f): \quad$ Jumps of $f$ (see (18))

$\delta_{n}^{s}\left(\theta, y_{n}\right)$ : Generalized finite difference (see (13)) $\delta_{n}^{s}\left(y_{n}\right)$ : Generalized finite difference with $\theta_{k}=1$, $k=1, \ldots, s$ (see Section 4 and the appendix)

$\Delta_{n}^{s}\left(y_{n}\right):$ Finite difference (see (A.1))

$B_{k}(x)$ : The Bernoulli polynomial (see (20))

$B_{n}(k)$ : The Fourier coefficient of the Bernoulli polynomial (see (21))

$\tau_{k}: \quad$ Some parameters joined to $\theta_{k}$ (see (32))

$\gamma_{s}(\tau)$ : Coefficients of polynomial with the roots $-1 / \tau_{k}($ see $(35))$.

\section{Acknowledgment}

The author acknowledges the reviewers and the editors for their valuable suggestions and constructive comments that helped to improve the paper.

\section{References}

[1] A. Poghosyan, "Asymptotic behavior of the Krylov-Lanczos interpolation," Analysis and Applications, vol. 7, no. 2, pp. 199211, 2009

[2] A. Krylov, On Approximate Calculations. Lectures Delivered in 1906, Tipolitography of Birkenfeld, St. Petersburg, Russia, 1907.

[3] C. Lanczos, "Evaluation of noisy data," Journal of the Society for Industrial and Applied Mathematics, vol. 1, pp. 76-85, 1964.

[4] C. Lanczos, Discourse on Fourier Series, Oliver and Boyd, Edinburgh, UK, 1966.

[5] B. Adcock, Modified Fourier expansions: theory, construction and applications [Ph.D. thesis], University of Cambridge, Trinity Hall, UK, 2010.

[6] G. Baszenski, F.-J. Delvos, and M. Tasche, "A united approach to accelerating trigonometric expansions," Computers \& Mathematics with Applications, vol. 30, no. 3-6, pp. 33-49, 1995.

[7] D. Batenkov and Y. Yomdin, "Algebraic Fourier reconstruction of piecewise smooth functions," Mathematics of Computation, vol. 81, no. 277, pp. 277-318, 2012.

[8] J. P. Boyd, "Acceleration of algebraically-converging Fourier series when the coefficients have series in powers in $1 / n$," Journal of Computational Physics, vol. 228, no. 5, pp. 1404-1411, 2009.

[9] A. Poghosyan, "Asymptotic behavior of the Eckhoff method for convergence acceleration of trigonometric interpolation," Analysis in Theory and Applications, vol. 26, no. 3, pp. 236-260, 2010.

[10] A. Poghosyan, "On an autocorrection phenomenon of the Eckhoff interpolation," The Australian Journal of Mathematical Analysis and Applications, vol. 9, no. 1, article 19, pp. 1-31, 2012.

[11] G. A. Baker and P. Graves-Morris, Pade Approximants, Encyclopedia of Mathematics and Its Applications, vol. 59, Cambridge University Press, Cambridge, UK, 2nd edition, 1966.

[12] E. W. Cheney, Introduction to Approximation Theory, McGrawHill, New York, NY, USA, 1966.

[13] J. F. Geer, "Rational trigonometric approximations using Fourier series partial sums," Journal of Scientific Computing, vol. 10, no. 3, pp. 325-356, 1995.

[14] A. Nersessian and A. Poghosyan, "On a rational linear approximation of Fourier series for smooth functions," Journal of Scientific Computing, vol. 26, no. 1, pp. 111-125, 2006. 
[15] A. Poghosyan, "On a convergence acceleration of trigonometric interpolation," Reports of the National Academy of Sciences of Armenia, vol. 112, no. 4, pp. 341-349, 2012.

[16] A. Poghosyan, A. Barkhudaryan, and S. Mkrtchyan, "Accelerating the convergence of trigonometric interpolation," in Proceedings of the 3rd Russian-Armenian Workshop on Mathematical Physics, Complex Analysis and Related Topics,, pp. 133-137, Tsaghkadzor, Armenia, 2010.

[17] A. Poghosyan, T. Barkhudaryan, and A. Nurbekyan, "Convergence acceleration of Fourier series by the roots of the Laguerre polynomial," in Proceedings of the 3rd Russian-Armenian Workshop on Mathematical Physics, Complex Analysis and Related Topics, pp. 138-142, Tsaghkadzor, Armenia, October 2010.

[18] A. V. Pogosyan, "On a linear rational-trigonometric interpolation of smooth functions," Reports of the National Academy of Sciences of Armenia, vol. 106, no. 1, pp. 13-20, 2006. 


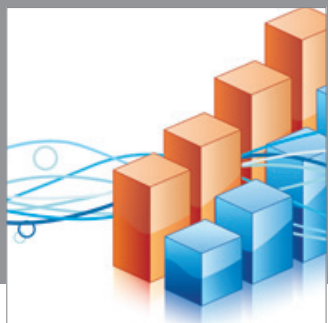

Advances in

Operations Research

mansans

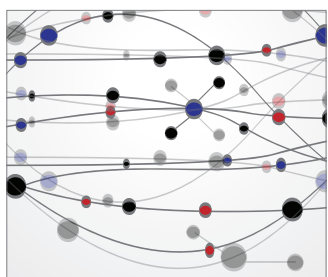

The Scientific World Journal
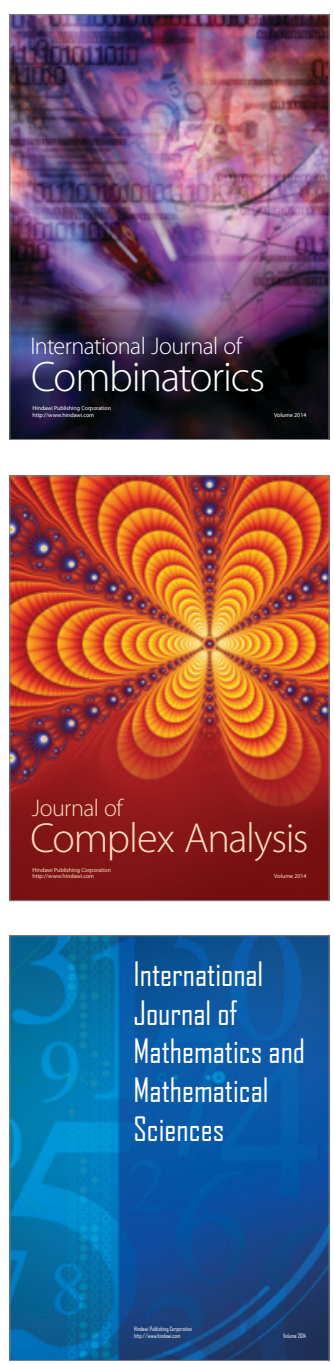
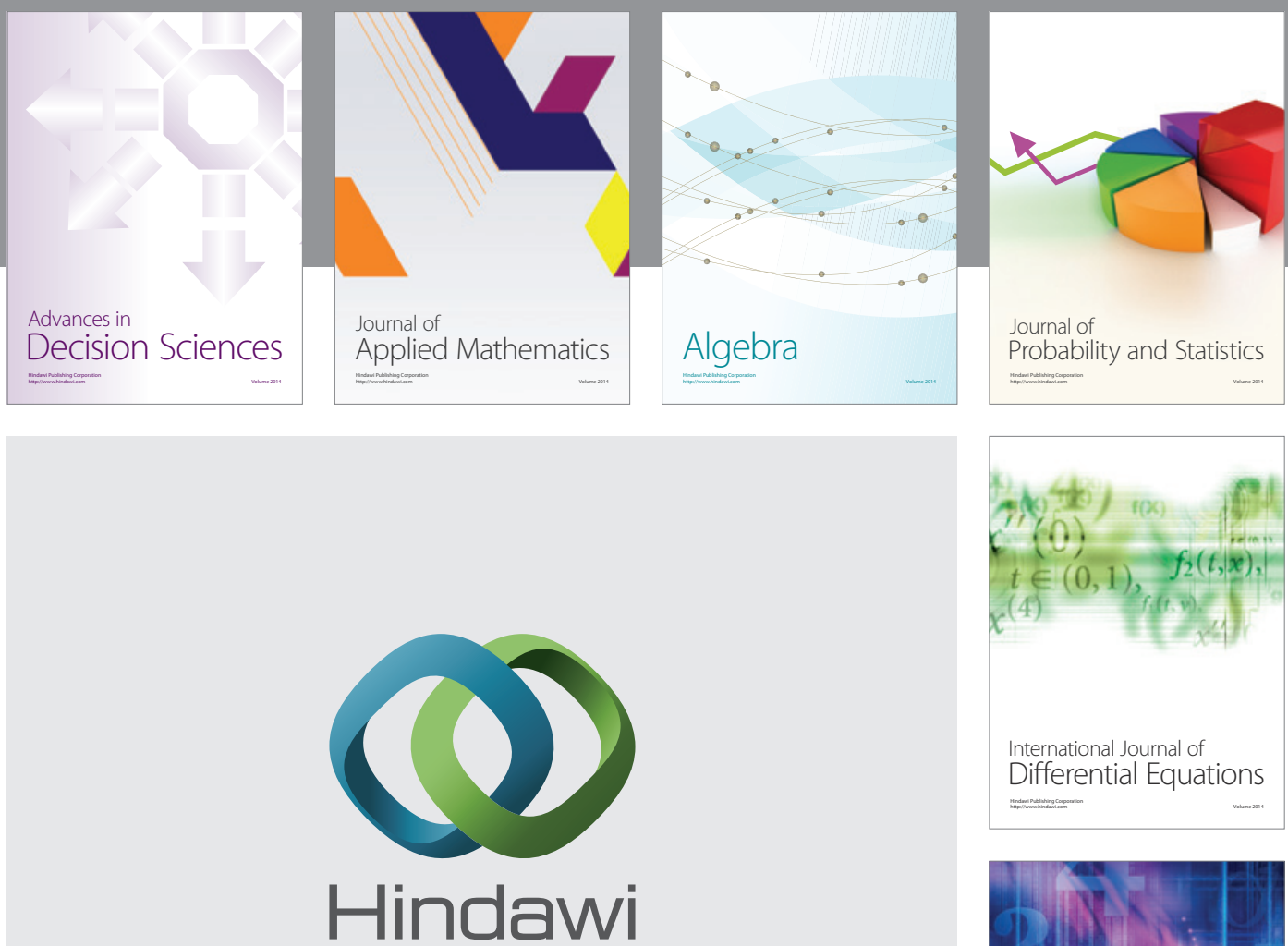

Submit your manuscripts at http://www.hindawi.com
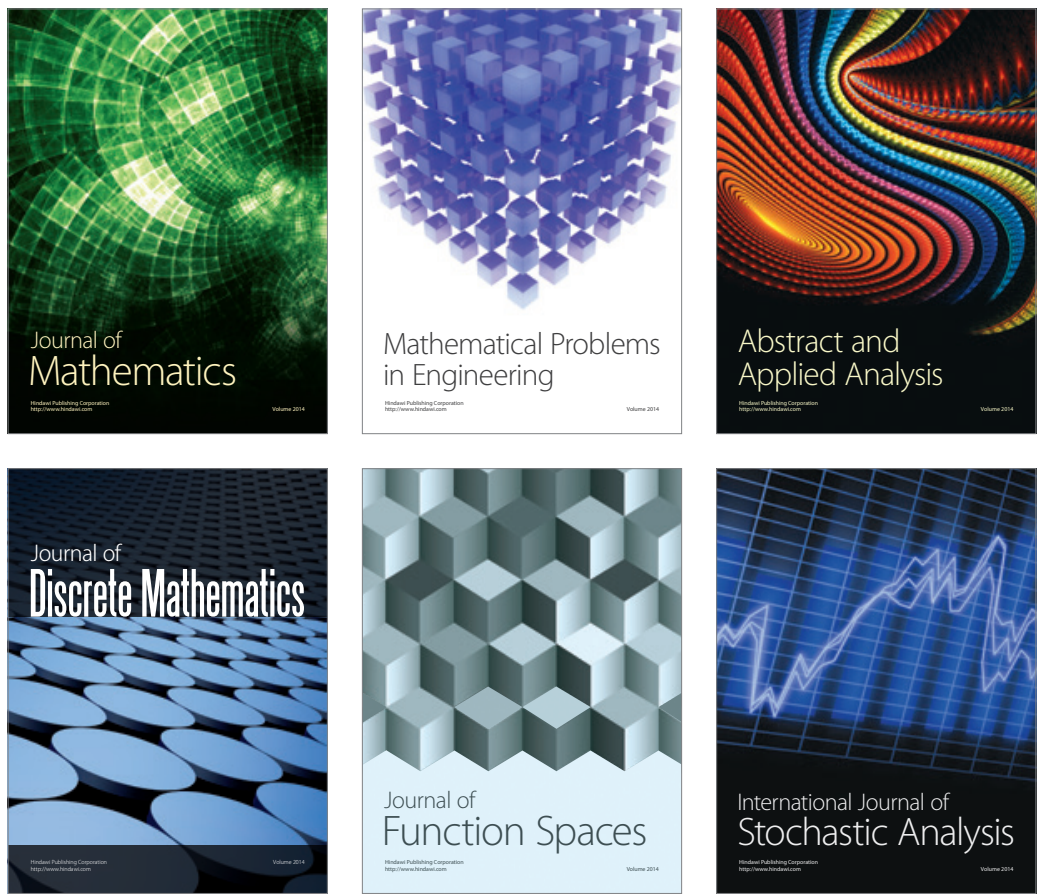

Journal of

Function Spaces

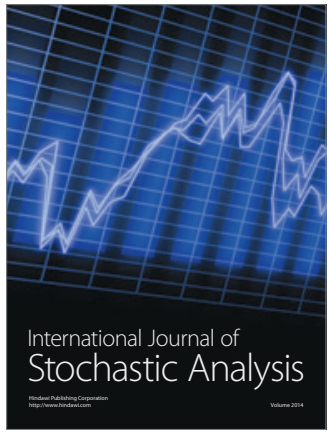

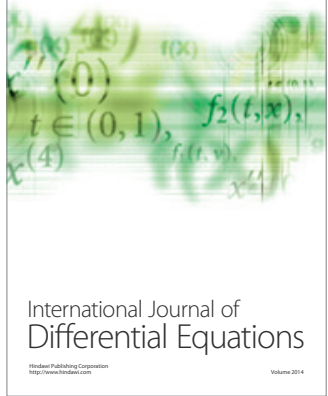
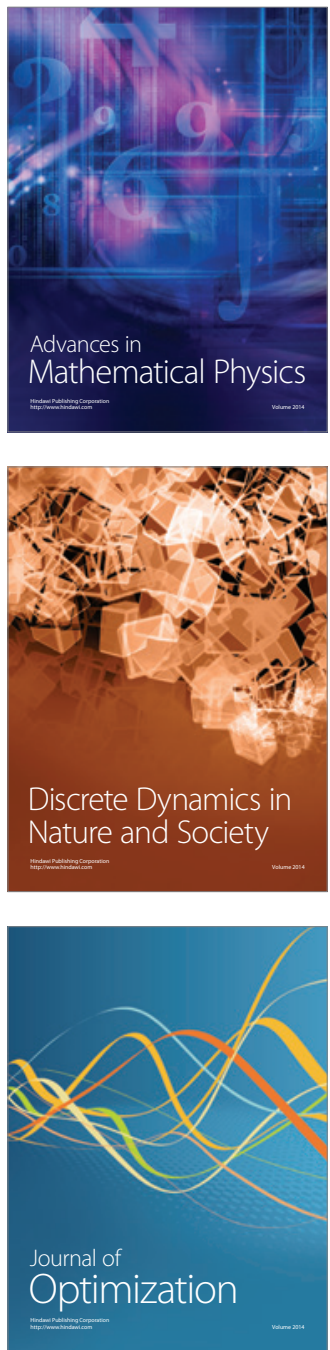